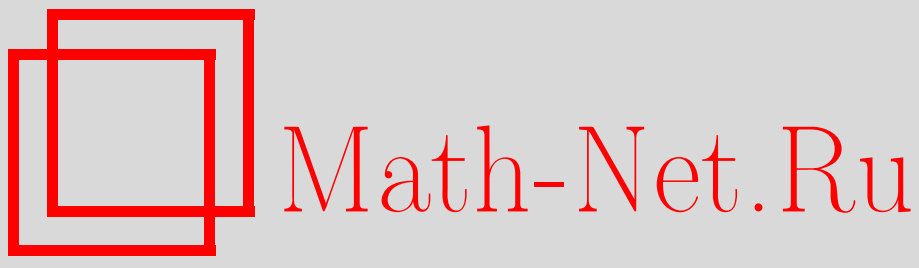

М. И. Зеликин, Л. Ф. Зеликина, Структура оптимального синтеза в окрестности особых многообразий для аффинных по управлению задач, Матем. сб., 1998, том 189, номер 10, 33-52

DOI: https://doi.org/10.4213/sm358

Использование Общероссийского математического портала Math-Net.Ru подразумевает, что вы прочитали и согласны с пользовательским соглашением

http://www.mathnet.ru/rus/agreement

Параметры загрузки:

IP: 52.90 .164 .192

26 апреля 2023 г., 16:02:48 
УДК 517.977

\author{
М.И. Зеликин, Л.Ф. Зеликина
}

\title{
Структура оптимального синтеза в окрестности особьх многообразий для аффинных по управлению задач
}

\begin{abstract}
Статья посвящена вопросам классификации фазовых портретов оптимального синтеза для аффинных по управлению систем постоянного ранга в окрестности особых универсальных многообразий. Как фазовое состояние, так и управление предполагаются многомерными. В основу классификации положен порядок особых экстремалей, а также свойства инволютивности или неинволютивности индикатрисы скоростей. Показано, что синтез оптимальных траекторий представляет собой расслоенное пространство над базой $W$, состоящей из особых оптимальных траекторий; слои состоят из неособых оптимальных траекторий. В случае многомерного управления особое многообразие $W$ является стратифицированным многообразием. В инволютивном случае слои одномерны. В неинволютивном случае слои многомерны и содержат траектории с учащающимися переключениями (chattering-траектории); размерность слоев и структура поля траекторий внутри этих слоев зависят от порядка особых экстремалей.

Библиографоия: 24 названия.
\end{abstract}

\section{§1. Введение}

Построение оптимального синтеза - одна из основных задач теории оптимального управления. Под оптимальньм синтезом понимают управление, построенное по принципу обратной связи, когда оптимальное управление определяется как функция от текущего фазового состояния управляемой системы. Соответствующее поле оптимальных траекторий называют синтезом оптимальных траекторий или фазовым портретом оптимального синтеза. Отдельные двумерные примеры оптимального синтеза были построены еще до того, как был сформулирован принцип максимума Понтрягина [1], [2]. Попытки развить общую теорию оптимального синтеза начались сразу с момента создания принципа максимума Понтрягина. В частности, В.Г. Болтянским в [3] была предложена концепщия регулярного синтеза и высказано предположение, что картина регулярного синтеза является типичной для задач оптимального управления. Однако в работах П. Бруновского [4], Л.Ф. Зеликиной [5], И. Купки [6], М. И. Зеликина и В. Ф. Борисова [7] и др.

Работа первого автора выполнена при финансовой поддержке Российского фонда фундаментальных исследований (грант № 96-01-01360) и гранта государственной поддержки ведущих научных школ (№ 96-15-96072).

Работа второго автора вьполнена при финансовой поддержке Российского фонда фундаментальных исследований (гранты № 97-01-00135, № 98-01-00535). 
было показано, что аксиомы регулярного синтеза Болтянского не выполняются в ситуациях общего положения.

Классификация двумерных фазовых портретов оптимального регулярного синтеза для афффинных по управлению задач была предложена М. М. Байтманом в [8]; полная теория двумерного оптимального синтеза была построена Г. Зуссманом [9].

Метод построения синтеза оптимальных траекторий, состоящий в описании инвариантов оптимального синтеза, для многомерных инволютивных систем аффинных по многомерному управлению был предложен в работе Зеликиной [10], где был построен $n$-мерньй синтез оптимальных траекторий, содержащий стратифицированное универсальное многообразие, т.е. многообразие, притягивающее к себе окрестные оптимальные траектории.

Задача описания границы областей достижимости (волновых фронтов) является в определенном смысле двойственной к задаче построения оптимального синтеза. А именно, вектору оптимальной скорости в данной точке можно поставить в соответствне касательную плоскость к области достижимости в этой точке, т.е. (в случае гладкости функции Беллмана) вектор сопряженных фазовых переменных. К. Лобри [11], Х. Шетлер [12], А. Кренер [13], А. Аграчев-И. Купка-Ж. Готье [14] и др. изучали структуру областей достижимости за малое время для аффинных по скалярному управлению систем размерности 3 и 4 .

Теория оптимального синтеза для неинволютивных систем произвольной размерности со скалярньм управлением развивалась в работах Зеликина и Борисова [7] и [15]. В этих работах была выяснена роль траекторий с учащающимися переключениями (chattering-траекторий) в структуре оптимального синтеза. Траекториями с учащающимися переключениями называются такие траектории, которые имеют бесконечное число переключений на конечном интервале времени; переключения накапливаются к точке сопряжения с особой траекторией. Показано, что в окрестности особых траекторий порядка 2 оптимальньй синтез имеет структуру chattering-расслоения с кусочно-гладкими двумерными слоями, заполняемыми оптимальными траекториями с учащающимися переключениями (chattering-траекториями) над базой, состоящей из особых оптимальных траекторий.

В данной статье дается описание фазового портрета оптимального синтеза в зависимости от порядка особых траекторий как для случая одномерного, так и для случая многомерного управления в инволютивном и неинволютивном случаях.

\section{§2. Постановка задачи}

Рассматривается задача минимизации функционала

$$
J=\int_{0}^{T}(f(x)+F(x) u) d t
$$

на множестве решений аффинной по управлению системы, т.е. системы вида

$$
\dot{x}=g(x)+G(x) u, \quad u \in U \text {. }
$$

Рассматриваются решения системы (2), которые начинаются в точке $x(0)=x_{0}$, $x_{0} \in \Omega \subset M$, и кончаются в момент времени $T$ - первого достижения заданного 
терминального многообразия $B \subset \bar{\Omega}$, т.е. $x(T) \in B$. Значение $T$ может быть и фиксированным ( $T=\infty$ не исключается). Случай, когда $f(x)+F(x) u \equiv 1$, называется задачей бъстродействия.

Здесь фазовое состояние системы описывается точкой $x \in M$, где $M$ - гладкое $n$-мерное многообразие; $u \in U$ - управление, где множество $U \subset \mathbb{R}^{k}$ есть вьпуклый многогранник. В качестве допустимого управления рассматриваются измеримые функции $u(t)$, принимающие значения в множестве $U$; допустимыми траекториями называются абсолютно непрерывные функции $x\left(t, x_{0}\right)$, удовлетворяюшие начальному условию $x\left(0, x_{0}\right)=x_{0}$ и являюшиеся решениями системы $(2)$, в которую подставлено допустимое управление $u(t)$. Отображения $g: M \rightarrow T_{x} M$, $G: M \rightarrow \operatorname{Lin}\left(\mathbb{R}^{k}, T_{x} M\right), f: M \rightarrow \mathbb{R}, F: M \rightarrow \operatorname{Lin}\left(\mathbb{R}^{k}, \mathbb{R}\right)$ задаются гладкими функциями. Под гладкими функциями всюду в дальнейшем понимаются функции класса $C^{\infty}$, под $\operatorname{Lin}(A, B)$ - линейный оператор из $A$ в $B$.

Под синтезом оптимального управления в области $\Omega$ для терминального многообразия $B$ понимается такое управление $u=u(x)$, что при всех $x_{0} \in \Omega$ система

$$
\dot{x}=g(x)+G(x) u(x)
$$

имеет решения в смысле Филиппова [16] $x\left(t, x_{0}\right) \in \Omega$ при $0 \leqslant t \leqslant T\left(x_{0}\right)$, которые лежат в области $\Omega$ и попадают на терминальное многообразие $B$ в момент $T\left(x_{0}\right)$, т.е. $x\left(T\left(x_{0}\right), x_{0}\right) \in B$; значение функционала $J$ на каждом решении минимально в классе допустимых траекторий, начинающихся в точке $x_{0}$ и не покидающих область $\Omega$.

Множество $A(x) \subset T_{x}^{*} M$, определяемое правой частью управляемой системы (2), $A(x)=\{g(x)+G(x) u: u \in U\}$, называется индикатрисой скоростей в точке $x$.

Пусть $y=(x, \psi)$ - локальные координаты на кокасательном расслоении $T^{*} M$. Здесь $\psi$ - двойственная переменная, определяющая линейный функционал на касательном пространстве $T_{x} M$, значение которого на элементе $q \in T_{x} M$ будет обозначаться как обычное умножение через $\psi q$. Пусть, для определенности, время $T$ не фиксировано.

\section{ПРИНЦИП МАКСИМУМА ПОНТРЯГИНА [17].}

Пусть $\hat{x}(t)$ - оптимальная траектория, $\hat{u}(t)$ - соответствующее оптимальное управление. Составим функцию Понтрягина

$$
H(x, \psi, u)=\psi(g(x)+G(x) u)-\psi_{0}(f(x)+F(x) u)=H_{0}\left(y, \psi_{0}\right)+H_{1}\left(y, \psi_{0}\right) u .
$$

Тогда существует константа $\psi_{0} \geqslant 0$ и такая непрерывная функция $\hat{\psi}: t \mapsto T_{x(t)}^{*} M$ $\left(\left(\psi_{0}, \hat{\psi}(t)\right) \neq 0\right)$, что кривая $y(t)=(\hat{x}(t), \hat{\psi}(t))$ в кокасательном расслоении $T^{*} M$ является решением гамильтоновой системы уравнений

$$
\dot{y}=\operatorname{sgrad}_{y} H\left(y, \psi_{0}, \hat{u}(t)\right),
$$


где sgrad - косой градиент функции $H$. На этом решении выполняются условие максимума:

$$
\max _{u \in U} H\left(y, \psi_{0}, u\right)=H\left(y, \psi_{0}, \hat{u}(t)\right) \equiv 0,
$$

и условие трансверсальности:

$$
\hat{\psi}(T) q=0
$$

для любого $q \in T_{\hat{x}(T)} B$.

Кривая $y(t)$, удовлетворяюшая условиям (4) и (5), называется әкстремалью в расширенном фазовом пространстве $T^{*} M$; ее проекция $x(t)$ на фазовое пространство $M$ - экстремальной траекторией.

Тот факт, что данная задача является аффинной по управлению, дает возможность свести ее к линейной по управлению задаче [18], в которой параметризация траекторий может выбираться произвольно. Поэтому длина векторов индикатрисы скоростей $A(x)$ является несушественной, и надо рассмотреть на многообразии $M$ поле полиэдральных конусов $K(x) \subset T_{x} M$, направляющими которых служат индикатрисы $A(x)$.

Введем следующие обозначения. Образующие конуса $K(x)$ будут обозначаться через $\varphi_{i}(x), i=1, \ldots, r$. Пусть $N$ - мультииндекс, который нумерует подмножество множества индексов $K=\{1, \ldots, r\}$. Под гранњю $L_{N}(x)$ конуса $K(x)$, порожденной набором образующих $\varphi_{i}(x), i \in N$, понимается

$$
L_{N}(x)=\operatorname{Con}\left\{\varphi_{i}(x), i \in N\right\}=\left\{\sum_{i \in N} \lambda_{i} \varphi_{i}(x): \lambda_{i} \geqslant 0\right\} .
$$

Относительная внутренность ri $L_{N}(x)$ грани $L_{N}(x)$ определяется следующим образом:

$$
\operatorname{ri} L_{N}(x)=\left\{\sum_{i \in N} \lambda_{i} \varphi_{i}(x): \lambda_{i}>0\right\} .
$$

Распределение плоскостей, порожденное гранью $L_{N}(x)$, обозначается через

$$
\operatorname{Span} L_{N}(x)=\left\{\sum_{i \in N} \lambda_{i} \varphi_{i}(x): \lambda_{i} \in \mathbb{R}\right\} .
$$

Будем говорить, что отрезок траектории $x(t), t \in\left(t_{0}, t_{1}\right)$, отвечает грани $L_{N}$, если $\dot{x}(t) \in L_{N}(x(t))$ при $t \in\left(t_{0}, t_{1}\right)$.

При построении оптимального синтеза определяюшую роль играют особые экстремальные траектории.

ОПРЕДЕЛЕНИЕ. Участок траектории $x(t), t \in\left(t_{0}, t_{1}\right)$, в фазовом пространстве $M$ называется $L_{N}$-особы.м. если $\dot{x}(t) \in \operatorname{ri} L(x(t))$ при $t \in\left(t_{0}, t_{1}\right)$.

В дальнейшем будут изучаться регулярные решения уравнений принципа максимума Понтрягина, т.е. такие решения, для которых $\psi_{0} \neq 0$. Следовательно, в силу однородности можно считать, что $\psi_{0}=1$.

Структура оптимального синтеза в окрестности особых экстремальных траекторий существенно различается в двух случаях: когда индикатриса скоростей инволютивна и когда она неинволютивна. 


\section{§ 3. Структура оптимального синтеза для инволютивных систем}

3.1. Распределение плоскостей $\operatorname{Span} L_{N}(x)$ называется инволютивным, если коммутатор любой пары векторных полей, принадлежащих $\operatorname{Span} L_{N}(x)$, также принадлежит $\operatorname{Span} L_{N}(x)$. Условие инволютивности достаточно проверить для образующих грани $\operatorname{Span} L_{N}(x)$, т.е. достаточно убедиться, что для любых $\varphi_{i}(x), \varphi_{j}(x) \in L_{N}(x)$

$$
\left[\varphi_{i}(x), \varphi_{j}(x)\right] \in \operatorname{Span} L_{N}(x) .
$$

Здесь $\left[\varphi_{i}(x), \varphi_{j}(x)\right]$ - коммутатор векторов $\varphi_{i}(x), \varphi_{j}(x)$.

Для простоты изложения рассмотрим задачи быстродействия для управляемых систем с индикатрисой скоростей $A(x)$, являюшейся $n$-мерным симплексом. В этом случае система (2) имеет вид:

$$
\dot{x}=\sum_{i=1}^{n} u_{i} \varphi_{i}(x), \quad u \in U,
$$

где

$$
U=\left\{u=\left(u^{1}, \ldots, u^{n}\right): u^{i} \geqslant 0, \sum_{i=1}^{n} u_{i}=1\right\} .
$$

В данной статье рассматриваются системы, для которых выполняется следующее предположение постоянства ранга.

ПРЕДПОЛОЖЕНИЕ 1. Векторные поля $\left\{\varphi_{i}(x)\right\}$ линейно независимы в облас$m u \Omega$.

Матрицу, столбцами которой являются векторы $\left\{\varphi_{i}(x)\right\}$, обозначим через $\Phi(x)=\left\|\varphi_{1}(x), \ldots, \varphi_{n}(x)\right\|$. При выполнении предположения 1 матрица $\Phi(x)$ невырождена.

ПРЕДПОЛОЖЕНИЕ 2. Для любого набора индексов $\left\{i_{1}, \ldots, i_{k}\right\}$ линейные оболочки векторных полей $\operatorname{Span}\left\{\varphi_{i_{1}}, \ldots, \varphi_{i_{k}}\right\}$ образуют инволютивнье распределения $k$-мерных плоскостей в $\Omega$.

Рассмотрим инволютивную грань $L_{I}, I=\left\{i_{1}, \ldots, i_{k}\right\}$, конуса $K(x)$. Пусть $\{\mathscr{L}\}$ - слоение, отвечающее распределению $\operatorname{Span} L_{I}$. В [17] показано, что по набору образующих конуса $L_{I}$, которые составляют базис пространства $\operatorname{Span} L_{I}$, можно построить такую дифференциальную форму $\omega$, что функционал (1) совпадает с интегралом от дифференциальной формы $\omega$ по траекториям системы $(2)$, принадлежащим грани $L_{I}$ и, следовательно, лежащим в слое $\mathscr{L}_{x(0)}$.

В случае задачи быстродействия $\omega$ совпадает с дифференциальной формой $d t$, выраженной через фазовые координаты $x$, т.е. полученной исключением управляюших переменных из дифференциальных уравнений (2), ограниченных на $\operatorname{Span} L_{I}$. Нетрудно убедиться, что для системы (7) на грани максимальной размерности дифференциальная форма $\omega$ имеет вид:

$$
\omega=\sum_{i=1}^{n}\left(\Phi^{-1}\right)_{j}^{i}(x) d x^{j},
$$

где $\left(\Phi^{-1}\right)_{j}^{i}$ - элементы матрицы $\left(\Phi^{-1}\right)$. 
ЗАмечание 1 . В случае, когда многогранник $U$ не является симплексом, та же конструкция позволяет сопоставить каждой $(n-1)$-мерной грани $L_{N}$ многогранника $U$ свою дифференциальную форму $\omega_{N}$. При этом формы $\omega_{N_{1}}$ и $\omega_{N_{2}}$ совпадают на пересечении граней $L_{N_{1}} \cap L_{N_{2}}$.

Обозначим через $i_{L}^{*}$ оператор ограничения дифференциальных форм на грань $L$.

Теорема 1 [19]. Предположим, что распределение плоскостей $\operatorname{Span} L$ инволютивно. Тогда необходимым условием оптимальности L-особой на участке $\left(t_{0}, t_{1}\right)$ траектории $x(t)$ является равенство

$$
i_{L}^{*} d \omega_{x(t)}=0, \quad t \in\left(t_{0}, t_{1}\right)
$$

По теореме 1 для инволютивных систем постоянного ранга проекции $L_{i j}$-особых экстремалей на фазовое пространство $M$ лежат на многообразии $V_{i j}$, которое задается уравнением

$$
v_{i j}=d \omega\left(\varphi_{i} ; \varphi_{j}\right)=0 .
$$

ПрЕДПОЛОЖЕнИЕ 3. Дифференциальная форма $\omega$ вполне интегрируема, т.е. $\omega \wedge d \omega=0$.

Дифференциальная форма $\omega$ является вполне интегрируемой тогда и только тогда, когда она может быть записана в форме монома $\omega=P(x) d Q(x)$. Функция $1 / P(x)$ называется интегрирующим множителем формы $\omega$, а поверхности $Q(x)=$ Const - ее интегральными поверхностями.

ТЕОРема 2 [19]. Пусть управляемая система (7) инволютивна и форма (8) вполне интегрируема. Предположим, что для L-особой траектории $x(t)$, $t \in[0, T]$, ограничение интегрирующего множителя формы $i_{L}^{*} \omega$ на пересечение интегральной поверхности этой формы с листом $\mathscr{L}_{x(0)}$ слоения $\{\mathscr{L}\}$ достигает локального максимума в точках $x(t)$ равномерно по $t \in[0, T]$.

Тогда траектория $x(t)$ дает сильный минимум функиионалу $J$.

Пусть грань $L_{N_{1}}$ конуса $K$ порождена образуюшими $\varphi_{i}(x), i \in N_{1}$, а грань $L_{N_{2}}$ - образующими $\varphi_{j}(x), j \in N_{2}$, причем $N_{1} \cap N_{2}=\varnothing$.

ТЕОРема 3 [18]. Пусть управляемая система (7) инволютивна и для всех точек некоторой области $D \subset \Omega$ выполнены неравенства

$$
d \omega\left(\varphi_{i}(x) ; \varphi_{j}(x)\right)<0, \quad i \in N_{1}, \quad j \in N_{2}
$$

Тогда траектория, которая в точке $x_{0} \in D$ переключается с управления, соответствующего грани $L_{N_{2}}$, на управление, соответствующее грани $L_{N_{1}}$, неоптимальна.

При выполнении условий теоремы 3 мы будем говорить, что в области $D$ грань $L_{N_{1}}$ мажсорирует грань $L_{N_{2}}$.

УТВЕРЖДЕНИЕ 1. Если дифференциальная форма $\omega$ является вполне интегрируемой положительно определенной на конусе $K$, mо $V_{i k} \cap V_{k j} \subset V_{i j}$. 
ДокАЗАТЕЛЬСТво. Для вполне интегрируемой формы $\omega$ имеем

$$
\omega \wedge d \omega\left(\varphi_{i} ; \varphi_{j} ; \varphi_{k}\right)=0,
$$

т.е. $\omega\left(\varphi_{i}\right) d \omega\left(\varphi_{j} ; \varphi_{k}\right)+\omega\left(\varphi_{j}\right) d \omega\left(\varphi_{k} ; \varphi_{i}\right)+\omega\left(\varphi_{k}\right) d \omega\left(\varphi_{i} ; \varphi_{j}\right)=0$. Из теоремы 1 следует, что в точке $x \in V_{i k} \cap V_{k j}$ первые два слагаемых левой части последнего равенства равны нулю. Поскольку $\omega\left(\varphi_{k}\right)>0$, имеем $d \omega\left(\varphi_{i} ; \varphi_{j}\right)=0$, т.е. $x \in V_{i j}$, что и требовалось доказать.

УТВеРЖДЕНИЕ 2 [19]. Пусть $L_{I}-k$-мерная грань конуса $K$ для инволютивной системы (7) постоянного ранга с вполне интегрируемой формой (8). Тогда для открытого, всюду плотного (в топологии Уитни) множества в пространстве дифференииальных форм, вполне интегрируемых и положительно определенных на конусе $K$, многообразие $V_{I}$, задаваемое системой уравнений

$$
v_{i j}=0, \quad i, j \in I,
$$

является гладким $(n-k+1)$-мерныцм многообразием или пустым множеством.

ДокАЗАТЕЛЬСтво. Фиксируем произвольный индекс $\alpha \in I$. Тогда в силу утверждения 1 из системы, состояшей из $(k-1)$ уравнений $v_{i \alpha}=0, i \in I, i \neq \alpha$, следуют все остальные уравнения системы (11). Докажем, что эти уравнения независимы. Для вполне интегрируемой формы $\omega=P(x) d Q(x)$ условие (11) эквивалентно условию пропорциональности проекций градиентов функций $P$ и $Q$ на грань $L_{I}$. Поскольку распределение $\operatorname{Span} L_{I}$ инволютивно, можно выбрать локальные координаты $x=\left(x_{1}, \ldots, x_{n}\right)$ в $\Omega$ так, чтобы грань $\operatorname{Span} L_{I}$ задавалась уравнениями $x_{k+1}=C_{k+1}, \ldots, x_{n}=C_{n}$. Обозначим через $J_{1}$ расслоение 1 -джетов отображений $\Omega \rightarrow \mathbb{R}^{2}$. Рассмотрим отображение $\theta: \Omega \mapsto(P(x), Q(x)) \in \mathbb{R}^{2}$ и его подъем в расслоение джетов $\Theta: \Omega \rightarrow J_{1}$. Пусть $p_{1}, \ldots, p_{n}, q_{1}, \ldots, q_{n}-$ координаты в слое $J_{1}$ над точкой $x$, отвечающие частным производным функций $P$ и $Q$, соответственно. Рассмотрим в $J_{1}$ многообразие $F$, задаваемое системой уравнений $p_{1} q_{i}=p_{i} q_{1}$, $i=2, \ldots, k$. Многообразие $V_{I}$ есть прообраз $F$ при отображении $\Theta$. Для завершения доказательства остается применить теорему трансверсальности Тома [18].

Перейдем к описанию универсальной структуры оптимального синтеза.

ОПРЕДЕЛЕНИЕ. Множество $W \subset \Omega$ в фазовом пространстве $M$ называется универсальным для некоторого синтеза $u(x)$, если для любой точки $x_{0} \in W$ существует $T>0$ такое, что для любого $\tau \in[0, T]$ сушествует такая окрестность $U_{x_{0}}$ точки $x_{0}$, что для любого $x \in U_{x_{0}}$ траектория синтеза, исходящая из точки $x$, в момент времени $\tau$ окажется на множестве $W$.

Из определения следует, что все траектории синтеза, находящиеся в некоторой окрестности универсального множества, выходят на него и далее движутся по немy.

Пусть $I=\left\{i_{1}, \ldots, i_{k}\right\}$ - определяет некоторую грань симплекса $U$. По теореме $1 L_{i j}$-особые оптимальные траектории должны лежать на многообразии, задаваемом уравнением $d \omega\left(\varphi_{i} ; \varphi_{j}\right)=0$. По теореме 3 для построения универсального 
оптимального синтеза надо использовать не все многообразие $V_{i j}$, а только ту его часть, которая задается следуюшими условиями:

$$
W_{I}=\left\{x \in M: d \omega\left(\varphi_{i} ; \varphi_{j}\right)=0, i, j \in I ; d \omega\left(\varphi_{i} ; \varphi_{j}\right)<0, i \in I, j \notin I\right\}
$$

Через $W$ обозначим множество

$$
W=\bigcup_{I} W_{I}
$$

В силу утверждений 1 и 2 стратифицированное многообразие $W$ топологически эквивалентно нерву многогранника $U$. А именно, каждой $k$-мерной грани многогранника $U$ отвечает (в силу утверждения 2$)(n-k)$-мерное особое многообразие; все страты $W_{J}$, отвечающие граням, принадлежащим границе любой фиксированной грани $L_{I}$, пересекаются по страту $W_{I}$. В частности, все страты $W_{I}$ пересекаются по одномерному страту $W_{K}$, отвечающему грани максимальной размерности.

Рассмотрим матрицу

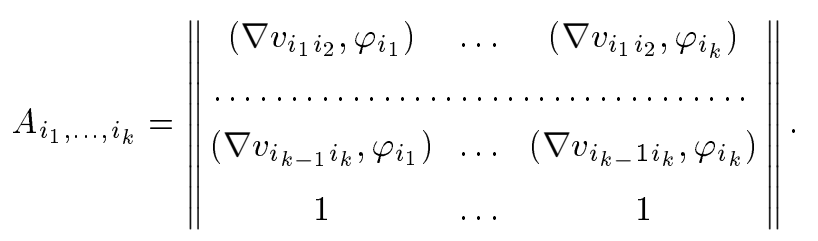

Введем обозначения $\Delta_{i_{1}, \ldots, i_{k}}=\operatorname{det} A_{i_{1}, \ldots, i_{k}}$. Алгебраическое дополнение к $j$-му элементу последней строки матрицы $A_{i_{1}, \ldots, i_{k}}$ обозначим через $\Delta_{i_{1}, \ldots, i_{k}}^{j}$.

ПРЕДПОЛОЖЕНИЕ 4. Для любого набора индексов $I=\left\{i_{1}, \ldots, i_{k}\right\}$ и для всех $j \in I$ выполнены неравенства $\Delta_{i_{1}, \ldots, i_{k}}^{j}>0$.

Рассмотрим следующий синтез $u^{*}(x)$.

В области $V_{\alpha}=\left\{x: v_{\alpha i}(x)<0, i \neq \alpha\right\}$ положим

$$
u^{*}(x)=e_{\alpha},
$$

где $e_{\alpha}$ - вершина многогранника $U$. При $x \in W_{i_{1}, \ldots, i_{k}}$ положим

$$
u_{j}^{*}(x)=\frac{\Delta_{i_{1}, \ldots, i_{k}}^{j}}{\Delta_{i_{1}, \ldots, i_{k}}}
$$




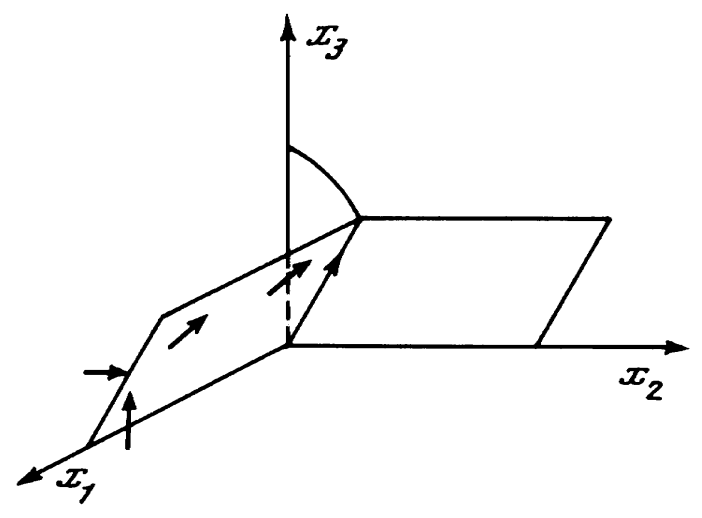

Рис. 1

ТЕОРема 4. Пусть для системы (7) выполнены предполодсения 1-4. Тогда $W=\bigcup_{I} W_{I}$ - стратифицированное многообразие, состоящее из особых әкстремалей. Страты $W_{I}$ являются универсальными множествами для синтеза (14), (15).

В случае, когда $\varphi_{i}(x)$ - координатные орты, теорема 4 была доказана в [10]. Доказательство общего случая будет приведено в последующих публикациях. Для $n=3$ многообразие $W$ показано на рис. 1 .

Отметим, что предположение 4 обеспечивает вьполнение теоремы 2 , дающей достаточные условия оптимальности особых траекторий.

3.2. Описание оптимальных траекторий в окрестности многообразия $W_{K}$. Оптимальный синтез $(14),(15)$ дает следующий фазовый портрет оптимальных траекторий в окрестности универсального многообразия $W_{K}$.

Стратифицированное многообразие $W$ разбивает окрестность $W_{K}$ на $n$ областей $V_{\alpha}$, в которых используется управление $e_{\alpha}$. Соответствующие оптимальные траектории достигают одного из стратов $W_{I}$ многообразия $W$, переключаются на управление (15), соответствуюшее этому страту, движутся по нему до пересечения с одним из стратов $W_{J} \subset \partial W_{I}$, переключаются на управление, соответствующее страту $W_{J}$, и продолжают таким же образом, последовательно переходя со стратов большей размерности на страты меньшей размерности, до тех пор, пока не достигнут $W_{K}$. Далее движение происходит по $W_{K}$.

Одномерньй страт $W_{K}$ играет роль магистрали в задаче оптимального быстродействия: большая часть траектории наибыстрейшего достижения достаточно удаленного терминального многообразия $B$ лежит на $W_{K}$.

\section{§4. Неинволютивная индикатриса скоростей. Одномерное управление}

4.1. Рассмотрим случай скалярного управления, т.е. случай, когда конус $K(x)$ является двумерным. Будем предполагать, что его образующие $\varphi_{i}(x), i=1,2$, 
линейно независимы в каждой точке области $\Omega$. Функция Понтрягина имеет вид: $H_{0}(y)+H_{1}(y) u$. На особой экстремали

$$
H_{1}(y(t))=0
$$

при всех $t \in\left(t_{0}, t_{1}\right)$. Для того чтобы найти управление на особой траектории, надо дифференцировать равенство (16) по $t$ в силу системы (4) до тех пор, пока в выражении для соответствующей производной не появится управление $u(t)$ с ненулевым коэффициентом. В результате первого дифференцирования получается скобка Пуассона функции Понтрягина системы $(4) H_{0}(y)+H_{1}(y) u$ и функции $H_{1}(y)$ :

$$
\left\{H_{0}, H_{1}\right\}=0 \text {. }
$$

Уравнение (17) не содержит управления и его надо продифференцировать еше раз. Получаем

$$
\left\{H_{0},\left\{H_{0}, H_{1}\right\}\right\}+u\left\{H_{1},\left\{H_{0}, H_{1}\right\}\right\}=0 .
$$

Если коэффициент $\left\{H_{1},\left\{H_{0}, H_{1}\right\}\right\}(y(t)) \equiv 0$, то дифференцирование следует продолжать. Известно [21], что первый отличный от нуля порядок производной, для которой коэффициент при управлении отличен от нуля, является четным числом $2 q$. Следуя Х. Дж. Келли, Р.Е. Коппу, Х.Г. Мойеру [21] и Р.М. Льюису [22], дадим следуюшие два определения.

ОПРЕДЕЛЕНИЕ. Участок экстремали $y(t)$ называется траекторией локального порядка $q$, если на нем

$$
\frac{\partial}{\partial u} \frac{d^{k}}{d t^{k}} H_{1}(y(t))=0, \quad k=0,1, \ldots, 2 q-1, \quad \frac{\partial}{\partial u} \frac{d^{2 q}}{d t^{2 q}} H_{1}(y(t)) \neq 0 .
$$

ОПРЕДЕЛЕНИЕ. Если соотношения (19) выполнены тождественно при всех $y$ (а не только на траектории $y(t)$ ), то мы будем говорить, что глобальныцй (intrinsic) порядок задачи равен $q$.

Ясно, что глобальный порядок задачи не превосходит локального порядка любой экстремали данной задачи.

4.2. Структура оптимального синтеза в окрестности многообразия особых экстремалей второго порядка. Рассмотрим сначала случай, когда глобальный порядок задачи (1), (2) равен 2. Принцип максимума Понтрягина приводит к разрывной гамильтоновой системе

$$
\dot{y}=\operatorname{sgrad}_{y}\left(H_{0}(y)+u H_{1}(y)\right), \quad u=\operatorname{sgn} H_{1}(y) .
$$

Здесь $y=(\psi, x) \in T_{x}^{*} M$. Обозначим через $\operatorname{ad}_{H_{0}}$ оператор взятия скобки Пуассона с функцией $H_{0}$, в частности,

$$
\operatorname{ad}_{H_{0}} H_{1}=\sum_{i=1}^{n}\left(\frac{\partial H_{0}}{\partial \psi_{i}} \frac{\partial H_{1}}{\partial x_{i}}-\frac{\partial H_{0}}{\partial x_{i}} \frac{\partial H_{1}}{\partial \psi_{i}}\right) .
$$

Если глобальный порядок задачи равен 2, то на особой экстремали выполнены условия

$$
z_{i}=\operatorname{ad}_{H_{0}}^{i-1} H_{1}=0, \quad i=1, \ldots, 4
$$


ПРЕДПОЛОЖЕНИЕ 5. Функиии $z_{i}, i=1, \ldots, 4$, функционально независимы в области $\Omega$.

Предположение 5 означает, что якобиан, составленный из производных этих функций по переменным $y$, имеет ранг 4. Следовательно, в окрестности любой точки особой экстремали $y(t)$ можно дополнить функции $z(y)=\left(z_{1}, z_{2}, z_{3}, z_{4}\right)$ функциями $w(y)=\left(w_{1}, \ldots, w_{2 n-4}\right)$ так, чтобы якобиан $\left.\operatorname{det}(D(z, w) / D y)\right|_{y(t)} \neq 0$. B peзультате замены переменных $y \mapsto(z, w)$ система $(20)$ примет вид:

$$
\begin{aligned}
\dot{z}_{1}=z_{2}, \quad \dot{z}_{2}=z_{3}, \quad \dot{z}_{3}=z_{4}, \quad \dot{z}_{4} & =\alpha(z, w)+\beta(z, w) u, \\
\dot{w}=\gamma(z, w, u), \quad u & =\operatorname{sgn} z_{1},
\end{aligned}
$$

правые части которой терпят разрыв на гиперплоскости $S=\left\{(z, w): z_{1}=0\right\}$.

Опишем симметрии главной части системы уравнений (21).

Если функции $\alpha$ и $\beta$ не зависят от $z$, то первые четыре уравнения системы $(21)$ инвариантны относительно следующей однопараметрической групшы преобразований:

$$
g_{\varkappa}(z)=\left(\varkappa^{4} z_{1}, \varkappa^{3} z_{2}, \varkappa^{2} z_{3}, \varkappa z_{4}\right),
$$

которая переводит траекторию $z(t), w(t)$ в траекторию $g_{\varkappa}(z(t / \varkappa)), w(t / \varkappa)$ и управление $u(t)$ в $u(t / \varkappa)$. Группа $g_{\varkappa}$ определяет градуировку переменных $z_{i}$ : координата $z_{1}$ имеет вес 4 , координата $z_{2}-$ вес 3 , координата $z_{3}-$ вес 2 , координата $z_{4}-$ вес 1 ; координаты $w$ и управление $u$ имеют вес нуль.

В дальнейшем будут рассматриваться не только системы глобального порядка 2, но и системы глобального порядка 1 с особыми экстремалями второго порядка. В этом случае системы общего положения получаются, если добавить к правым частям системы (21) члены, имеющие в окрестности особой экстремали более высокий порядок малости по сравнению с соответствующей правой частью (в смысле вышеописанной градуировки):

$$
\begin{gathered}
\dot{z}_{1}=z_{2}+f_{1}(z, w, u), \quad \dot{z}_{2}=z_{3}+f_{2}(z, w, u), \quad \dot{z}_{3}=z_{4}+f_{3}(z, w, u), \\
\dot{z}_{4}=\alpha(w)+\beta(w) u+f_{4}(z, w, u), \quad \dot{w}=\gamma(z, w, u), \quad u=\operatorname{sgn} z_{1},
\end{gathered}
$$

где зависимость функций $\alpha$ и $\beta$ от $z$ включена в добавленный член $f_{4}$. Тот факт, что добавленные члены имеют более высокий порядок малости, выражается следующим соотношением, которое предполагается выполненным при всех $u \in U$ для некоторой области $D \subset T^{*} M$ :

$$
\limsup _{\varkappa \rightarrow+0} \varkappa^{i-5}\left\|f_{i}\left(g_{\varkappa} z, w, u\right)\right\|_{C(D)} \leqslant C
$$

где $C$ - некоторая положительная константа.

Для того чтобы произвести сведение, следует выбрать в качестве координат $z_{i}$ последовательные производные от функции $H_{1}$ (или выражения, которые отличаются от этих производных на величины высшего порядка в смысле градуировки $(22))$. Из (24) следует, что $f_{i}(0, w, u)=0, i=1, \ldots, 4$. Следовательно, в новых координатах семейство особых экстремалей заполняет плоскость $S_{0}=\{(z, w)$ : $z=0\}$. Коразмерность многообразия $S_{0}$ равна 4 . 
ТЕОРЕма 5 (О расслоении). Пусть для системь (23) в некоторой области $D$ выполнены следующие условия:

i) условие (24);

ii) $\beta<0$;

iii) $|\alpha|<-\beta$.

Тогда в некоторой окрестности $G$ множсества $D \cap S_{0}$ определень два расслоения $p^{+}: \mathfrak{N}^{+} \rightarrow S_{0}$ и $p^{-}: \mathfrak{N}^{-} \rightarrow S_{0}$ с кусочно-гладкими двумерными слоями $\mathfrak{N}_{\sigma}^{+}$и $\mathfrak{N}_{\sigma}^{-}$, соответственно. Кажсдый слой $\mathfrak{N}_{\sigma}^{+}$расслоения $\mathfrak{N}^{+}$является двумерным интегральным многообразием системы (23) и состоит из траекторий, которье счетное число раз пересекают поверхность разрыва S и за конечное время приходят в точку $\sigma$ поверхности $S_{0}$. Каэсдий слой $\mathfrak{N}_{\sigma}^{-}$расслоения $\mathfrak{N}^{-}$также является двумерным интегральным многообразием системы (23) и состоит из траекторий, которые счетное число раз пересекают поверхность разрыва $S$ и за конечное время приходят в точку $\sigma$ поверхности $S_{0}$ при регрессивном течении времени.

Диаграмма, приведенная на рис. 2, отражает расположение многообразий $\mathfrak{N}^{+}$ и $\mathfrak{N}^{-}$; при этом двумерные слои $\mathfrak{N}_{\sigma}^{+}$и $\mathfrak{N}_{\sigma}^{-}$изображены в виде одномерных кривых.

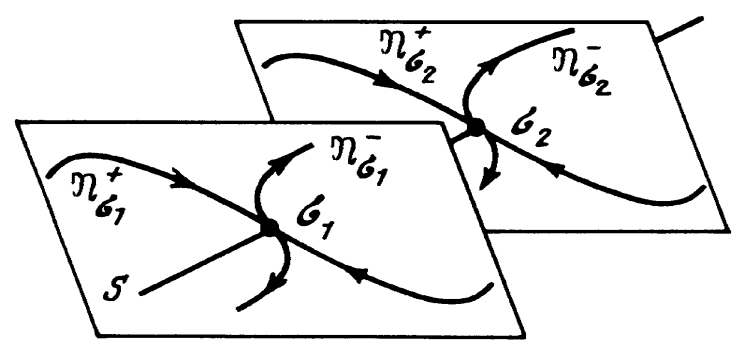

Рис. 2

Теорема 5 описывает (нестандартную) седловую структуру фазового портрета разрывной гамильтоновой системы в окрестности многообразия $S_{0}$ особых траекторий порядка 2. А именно, утверждается, что в окрестности многообразия $S_{0}$ существуют два $(2 n-2)$-мерных уса: устойчивый и неустойчивый. При этом $S_{0}=\mathfrak{N}^{+} \cap \mathfrak{N}^{-}$и окрестность многообразия $S_{0}$ гомеоморфна картине трансверсального пересечения многообразий $\mathfrak{N}^{+}$и $\mathfrak{N}^{-}$, так как

$$
\operatorname{dim} \mathfrak{N}^{+}+\operatorname{dim} \mathfrak{N}^{-}-\operatorname{dim} S_{0}=\operatorname{dim} T^{*} M
$$

Однако с точки зрения гладкой топологии усы $\mathfrak{N}^{+}$и $\mathfrak{N}^{-}$касаются друг друга, так что вьшеописанная структура является многомерньм аналогом сплюшенного седла. Траектории, начинающиеся в точках устойчивого уса, за конечное время со счетным числом переключений подходят к многообразию $S_{0}$ и далее идут по особой траектории, лежащей в этом многообразии. Та же самая картина, но с обращенным течением времени, имеет место в точках неустойчивого уса. 
Замечание 2. Условия теоремы 5 не могут быть усилены. А именно, если особая траектория имеет локальный порядок 2 , то $\beta(y(t)) \neq 0$. Необходимое условие оптимальности Лежандра-Клебша-Келли заключается в том, что $\beta \leqslant 0$, следовательно, $\beta<0$. Для того чтобы существовало управление $|u|<1$, реализующее движение по особым траекториям $y(t)$, необходимо, чтобы $|\alpha|<-\beta$.

Для того чтобы построить синтез оптимальных траекторий для задачи (1), (2), достаточно выделить в пространстве $T^{*} M$ лагранжево многообразие, регулярно проектируюшееся на многообразие $M$ (т.е. проекция должна быть диффеоморфизмом всюду, кроме точек переключения, которые могут быть точками негладкости лагранжева многообразия). Многообразие $\Lambda \subset T^{*} M(\operatorname{dim} \Lambda=n)$ называется $\Omega a$ гранжевым, если для любого кусочно-гладкого замкнутого контура $\gamma \subset \Lambda$ имеет место равенство

$$
\oint_{\gamma} \psi d x=0
$$

Многообразие $S_{0}$, состоящее из особых экстремалей, является $(2 n-4)$-мерным симплектическим многообразием и его лагранжевы подмногообразия имеют размерность $(n-2)$.

Теорема 6. Пусть $l \subset S_{0}$ - лагранжево подмногообразие многообразия $S_{0}$. Тогда полный прообраз l в расслоении $\mathfrak{N}^{+}$, т.е. $\left(p^{+}\right)^{-1}$, является лагранжевицм подмногообразием многообразия $T^{*} M$.

Доказательство теорем 5 и 6 основано на идее обобщенного $\sigma$-раздутия, т.е. на разрешении особенности отображения Пуанкаре поверхности переключения на себя. Полное доказательство приведено в [15] и [7].

Для описания оптимального синтеза для задачи (1), (2) в окрестности многообразия особых траекторий порядка 2 сформулируем результаты, касаюшиеся оптимального синтеза двумерной задачи Фуллера: минимизировать

при ограничениях

$$
\int_{0}^{\infty} x^{2} d t
$$

$$
\dot{x}=y, \quad \dot{y}=u, \quad|u| \leqslant 1 .
$$

Кривая переключения оптимального синтеза задается следуюшим уравнением: $x=-C y^{2} \operatorname{sgn} y$. Вьше кривой переключения $u=-1$, а ниже $-u=1$. Оптимальные траектории имеют вид закручивающихся к началу координат спиралей, которые пересекают поверхность переключения счетное число раз. Интервалы времени между последовательньми переключениями образуют убываюшую геометрическую прогрессию, и поэтому фазовая точка попадает в начало координат за конечное время.

Вернемся к задаче (1), (2). Обозначим через $\pi$ каноническую проекцию $\pi: T^{*} M \rightarrow M$. Если многообразие $\left(p^{+}\right)^{-1} l$ регулярно проектируется на $M$, то оно определяет расслоение $M$ на двумерные кусочно-гладкие слои $\pi\left(\mathfrak{N}_{\sigma}^{+}\right)$над базой $\pi(l)$. Фазовый портрет внутри каждого из слоев орбитально эквивалентен оптимальному синтезу двумерной задачи Фуллера.

Описанньй синтез полностью определяет универсальную структуру в окрестности многообразия, состоящего из особых траекторий локального порядка 2. 


\section{3. Структура оптимального синтеза в окрестности многообразия} особых экстремалей порядка $k$. В случаях особых экстремалей более высокого порядка результаты являются не столь полными. В работах [15] и [7] Зеликиньм сформулирована серия гипотез, обобшаюших вышеприведенные результаты на случай порядка особых экстремалей большего двух. Приведем здесь эти гипотезы.

По аналогии с классической двумерной задачей Фуллера можно сформулировать ее $k$-мерньй вариант: минимизировать

$$
\int_{0}^{\infty} x^{2} d t
$$

при ограничениях

$$
x^{(k)}=u, \quad \beta \leqslant u \leqslant 1,
$$

где $\beta<0$. Гамильтонова система принципа максимума Понтрягина для этой задачи имеет вид:

$$
\dot{z}_{i}=z_{i+1}, \quad i=1, \ldots,(2 k-1), \quad \dot{z}_{2 k}=\left\{\begin{array}{cc}
1 & \text { при }(-1)^{k} z_{1}<0 \\
\beta & \text { при }(-1)^{k} z_{1}>0 .
\end{array}\right.
$$

Легко видеть, что система (25) выдерживает группу диффееоморфизмов

$$
g_{\varkappa}: z_{i} \rightarrow \varkappa^{2 k+1-i} z_{i}, \quad i=1, \ldots, 2 k, \quad \varkappa>0,
$$

при которой траектория $z(t)$ переходит в $g_{\varkappa} z(t / \varkappa)$.

Первая группа гипотез относится к системе (25).

ГИПотеЗА 1. В фазовом пространстве $\mathbb{R}^{2 k}$ имеется два $k$-мерных, инвариантных относительно действия группы $g_{\varkappa}$ интегральных многообразия системы (25): $\mathfrak{N}^{+}$и $\mathfrak{N}^{-}$. Траектории системь (25), принадлежащие $\mathfrak{N}^{+}$, за конечное время подходят к началу координат; траектории системы (25), принадлежащие $\mathfrak{N}^{-}$, за конечное время подходят к началу координат при регрессивном течении времени.

Система (25) допускает понижение порядка с помощью факторизации по действию группы $g_{\varkappa}$. Профакторизованная система описывает эволюцию орбит группы $g_{\varkappa}$. Профакторизованную систему (25) будем обозначать через $(25)_{g}$; образы многообразий $\mathfrak{N}^{+}$и $\mathfrak{N}^{-}$- через $\mathfrak{N}_{g}^{+}$и $\mathfrak{N}_{g}^{-}$, соответственно.

ГиПОТеЗА 2. При $k=2 m$ внутри многообразия $\mathfrak{N}_{g}^{+}$имеется $m$ предельныц ииклов системы $(25)_{g}$, которые соответствуют однопараметрическим семействам автомодельных траекторий системы (25) с учащающимися переключениями, входящих за конечное время в начало координат. При $k=2 m+1$ у системы $(25)_{\text {g }}$ имеется $m$ предельных ииклов и еще два положения равновесия, которые соответствуют одномерным траекториям системы (25), каждая из которых входит в начало координат без переключения: одна $c$ $u=1$, а другая $с и=\beta$. Та же структура, но с обращением течения времени, имеет место внутри многообразия $\mathfrak{N}^{-}$. 
ГИПОТезА 3. Многообразия $\mathfrak{N}^{+}$u $\mathfrak{N}^{-}$вложены в $\mathbb{R}^{2 k}$ как кусочно-гладкие многообразия, пересекающие поверхность переключения $z_{1}=0$ по кусочно-гладкой поверхности, гомеоморфной $\mathbb{R}^{k-1}$.

Вторая группа гипотез относится к гамильтоновым системам, полученньм возмушением правых частей системы (25) слагаемыми более высокого порядка в смысле градуировки, порождаемой группой $g_{\varkappa}$ :

$$
\begin{gathered}
\dot{z}_{i}=z_{i+1}+f_{i}(z, w, u), \quad i=1, \ldots,(2 k-1), \\
\dot{z}_{2 k}=\alpha(w)+\beta(w) u+f_{2 k}(z, w, u), \\
\dot{w}=\gamma(z, w, u), \quad u=\operatorname{sgn} z_{1},
\end{gathered}
$$

где $w \in \mathbb{R}^{2 m}, z=\left(z_{1}, \ldots, z_{2 k}\right) \in \mathbb{R}^{2 k}$ и

$$
\limsup _{\varkappa \rightarrow+0} \varkappa^{2 k+1-i}\left\|f_{i}\left(g_{\varkappa} z, w, u\right)\right\|_{C(D)} \leqslant C,
$$

$D$ - некоторая область, содержашая часть плоскости $z=0$, в которой выполнены неравенства

$$
(-1)^{k} \beta(w)<0, \quad|\alpha(w)|<|\beta(w)| .
$$

Легко видеть, что многообразие $W=D \cap\{z=0\}$ состоит из особых траекторий локального порядка $k$.

ГиПотезА 4. В достаточно малой окрестности многообразия $W$ определены два кусочно-гладких расслоения $p^{+}: \mathfrak{N}^{+} \rightarrow W$ и $p^{-}: \mathfrak{N}^{-} \rightarrow W$ над базой $W$ и k-мерныцми кусочно-гладкими слоями $\mathfrak{N}_{w}^{+}$и $\mathfrak{N}_{w}^{-}$, соответственно, которые являются интегральньми многообразиями системы (27). Поток траекторий внутри каждого из многообразий $\mathfrak{N}_{w}^{ \pm}$орбитально әквивалентен потоку внутри многообразий $\mathfrak{N}^{ \pm}$, описанному в гипотезах $1-3$.

ГИПОТЕЗА 5. Если $L \subset W$ - лагранжево подмногообразие особого многообразия $W$, то $\left(p^{+}\right)^{-1} L$ и $\left(p^{-}\right)^{-1} L-$ лагранжсевы подмногообразия $\mathbb{R}^{2 k+2 m}$.

Гипотеза 1 в части, касаюшейся сушествования $n$-мерного устойчивого уса, доказана в [23]; доказательство гипотезы 2 получено в [24].

Подведем итог. Если проекция лагранжева многообразия $\left(p^{+}\right)^{-1} L$ на фазовое пространство регулярна, то она определяет локально оптимальный синтез, являющийся своеобразной декомпозицией двух систем, вообще говоря, не являющейся прямым произведением. Фазовое пространство расслаивается на $k$-мерные слои, состоящие из неособых оптимальных траекторий над многообразием особых траекторий $W$. При этом многообразие $W$ оказывается универсальным: сначала движение происходит внутри слоев; совершив счетное число переключений, точка за конечное время выходит на многообразие $W$. Потом она идет по особым траекториям внутри $W$. Фазовый портрет внутри каждого из слоев орбитально эквивалентен устойчивому усу $k$-мерной задачи Фуллера. 


\section{§5. Неинволютивная индикатриса скоростей. Многомерное управление}

В этом параграфе будет рассмотрен широкий класс неинволютивных аффинных по управлению систем с индикатрисой скоростей, являющейся симплексом.

Пусть $x=\left(x_{1}, \ldots, x_{n}\right) \in \mathbb{R}^{n}, u=\left(u_{1}, \ldots, u_{n}\right) \in U=\left\{u \in \mathbb{R}^{n}: \sum_{i=1}^{n} u_{i}=1\right.$, $\left.u_{i} \geqslant 0\right\}$, и $Q: \mathbb{R}^{n} \rightarrow \mathbb{R}^{1}$ - гладкая функция.

Рассмотрим задачу оптимального быстродействия на некоторое терминальное многообразие $B$ для управляемой системы

$$
x_{i}^{(k)}=u_{i} Q(x), \quad i=1, \ldots, n .
$$

Положим $\dot{x}=y_{1}, \ldots, x^{(k-1)}=y_{k-1}, y_{i} \in \mathbb{R}^{n}$. Для обозначения частных производных функции $Q$ будут использоваться нижние мультииндексы, например, $Q_{12}=\partial^{2} Q / \partial x_{1} \partial x_{2}$.

$\mathrm{C}$ помощью последовательного дифференцирования можно показать, что на $L_{12}$-особом экстремальном многообразии выполнена следуюшая система уравнений:

$$
\left\{\begin{array}{l}
Q_{1}-Q_{2}=0 \\
\sum_{i=1}^{n}\left(Q_{1 i}-Q_{2 i}\right)\left(y_{1}\right)_{i}=0 \\
\sum_{i, j=1}^{n}\left(Q_{1 i j}-Q_{2 i j}\right)\left(y_{1}\right)_{i}\left(y_{1}\right)_{j}+\sum_{i=1}^{n}\left(Q_{1 i}-Q_{2 i}\right)\left(y_{2}\right)_{i}=0 \\
\ldots \ldots \ldots \ldots \ldots \ldots \ldots \ldots \ldots \ldots \ldots \ldots \ldots \ldots \ldots \ldots \ldots \ldots \ldots \ldots \ldots \ldots \ldots \\
P_{k-1}(x, y)+\sum_{i=1}^{n}\left(Q_{1 i}-Q_{2 i}\right)\left(y_{k-1}\right)_{i}=0 .
\end{array}\right.
$$

Наконец, производная порядка $2 k$ дает

$$
P_{k}(x, y)+\sum_{i=1}^{n}\left(Q_{1 i}-Q_{2 i}\right) u_{i} Q(x)=0 .
$$

Здесь через $P_{i}(x, y)$ обозначены полиномиальные по $y$ функции, коэффициенты которых выражаются через частные производные от функции $Q(x)$. Ненулевой коэффициент при управлении $u$ возникает на $(2 k)$-м шаге дифференцирования, поэтому глобальный порядок задачи равен $k$.

Уравнения (29) определяют $(n-1) k$-мерное многообразие $W_{12}$, на котором лежат все $L_{12}$-особые экстремальные траектории, $L_{I}$-особые экстремальные траектории лежат на многообразии

$$
W_{I}=\bigcap_{i, j \in I} W_{i j}
$$

Страты $W_{i j}$ стратифицированного многообразия

$$
W=\bigcup_{I} W_{I}
$$


будем называть основными , так как в силу формулы (30) все остальные страты многообразия $W$ получаются при их пересечении. Отличие многообразия $W$ от того, которое было построено в $\S 3$ для инволютивного случая, состоит в том, что коразмерность любого основного страта (в ситуации общего положения) равна $k$ (а не 1, как в инволютивном случае), а коразмерность пересечения двух основных стратов равна $2 k$. Этот факт открывает возможность перехода со страта $W_{I}$ на страт $W_{J}$ меньшей размерности не с помощью кусочно-непрерьвного управления (как в инволютивном случае), а в режиме учашающихся переключений.

Для облегчения дальнейшего изложения разберем подробнее простейший из нетривиальных случаев $k=2, n=3$. Пусть, для конкретности, $Q(x)=x_{1} x_{2} x_{3}$, а область $\Omega=\mathbb{R}_{+}^{6}$. В этом случае гамильтонова система принципа максимума Понтрягина имеет вид:

$$
\left\{\begin{array}{l}
\dot{x}=y \\
\dot{y}=u x_{1} x_{2} x_{3}, \\
\dot{\psi}_{x_{i}}=-\psi u \frac{\partial\left(x_{1} x_{2} x_{3}\right)}{\partial x_{i}}, \\
\dot{\psi}_{y}=-\psi_{x},
\end{array}\right.
$$

где управление $u$ определяется из условия

$$
\max _{u \in U} \psi_{y} u
$$

Легко видеть, что любая допустимая траектория, начинающаяся в области $\Omega$, остается в $\Omega$ при всех $t>0$. Основные страты особого многообразия $W$ задаются уравнениями

$$
W_{i j}=\left\{x_{i}=x_{j}, y_{i}=y_{j}\right\} .
$$

Их пересечением является страт

$$
W_{123}=\left\{x_{1}=x_{2}=x_{3}, y_{1}=y_{2}=y_{3}\right\}
$$

Ограничение функции $x_{1} x_{2} x_{3}$ на плоскость $x_{1}+x_{2}+x_{3}=$ Const достигает максимума в точке $x_{1}=x_{2}=x_{3}$. Поэтому с точки зрения скорости роста координат векторов $x$ и $y$ наиболее выгодно двигаться вдоль многообразия $W_{123}$. Пусть сначала траектория выходит на основной страт, скажем, на $W_{12}$, чередуя управления $\left(u_{1}=1, u_{2}=u_{3}=0\right)$ и $\left(u_{2}=1, u_{1}=u_{3}=0\right)$, а затем, переключившись на особое управление $\left(u_{1}=u_{2}=\frac{1}{2}, u_{3}=0\right)$, мы хотим попасть на $W_{123}$.

УТВеРЖДЕНИЕ 3. Оптимальная $L_{123}$-особая траектория не может сопрягаться с кусочно-гладкой траекторией (как неособой, так и особой по паре индексов).

В силу утверждения 3 для достижения $W_{123}$ необходимо использовать режим учащающихся переключений. При этом единственной альтернативой управлению $\left(u_{1}=u_{2}=\frac{1}{2}, u_{3}=0\right)$, при котором а priory можно было бы оставаться в $W_{12}$, является управление $\left(u_{3}=1, u_{1}=u_{2}=0\right)$. 
УТВЕРЖДЕНИЕ 4. Страт $W_{12}$ является интегральным многообразием гамильтоновой системь (31), в которую подставлено управление $\left(u_{3}=1\right.$, $\left.u_{1}=u_{2}=0\right)$.

Справедливость утверждения 4 проверяется прямым дифференцированием. У верждение 4 показывает, что управление, на котором чередуются точки $a=\left(u_{1}=\right.$ $\left.u_{2}=0, u_{3}=1\right)$ и $b=\left(u_{1}=u_{2}=\frac{1}{2}, u_{3}=0\right)$, удерживает траекторию внутри страта $W_{12}$. Ограничив управление на отрезок, соединяющий точки $a$ и $b$, мы получим вспомогательную задачу с одномерным управлением, к которой применимы теоремы 5 и 6 . Следовательно, взяв произвольное потенциальное поле $\psi_{x}, \psi_{y}$ на многообразии $W_{123}$ (соответствуюшее лагранжево многообразиевспомогательной задачи определено неоднозначно и зависит от выбора терминального многообразия $B$ ), можно построить расслоение $\mathfrak{M}^{+}: W_{12} \rightarrow W_{123}$ с двумерными слоями $\mathfrak{M}_{\sigma}^{+}$, составленными из траекторий с учащающимися переключениями, чередующими управления $u=a$ и $u=b$. В силу теоремы 6 поле $\psi_{x}, \psi_{y}$ продолжается на страт $W_{12}$, что определяет лагранжево многообразие в расширенном фазовом пространстве над $W_{12}$ и дает возможность построить расслоение $\mathfrak{N}^{+}$окрестности страта $W_{12}$ над базой $W_{12}$, в слоях которого чередуются управления $u_{1}=1$ и $u_{2}=1$.

Итак, оптимальные траектории из достаточно малой окрестности $V_{12}$ основного страта $W_{12}$ сначала выходят на $W_{12}$ за конечное время с бесконечньм числом переключений, чередуюшихся управлений $u_{1}=1$ и $u_{2}=1$, а затем, оставаясь в $W_{12}$, выходят на $W_{123}$ за конечное время с бесконечным числом переключений, чередующих $u=a$ и $u=b$.

На рис. 3 кривые $S_{12}, S_{13}, S_{23}$ представляют четырехмерные многообразия $W_{12}, W_{13}, W_{23}$, соответственно; точка $S_{123}$ представляет двумерное многообразие $W_{123}$. Размеры окрестности $V_{i j}$ уменьшаются по мере приближения к страту $W_{123}$ и в точках этого страта сводятся к нулю.

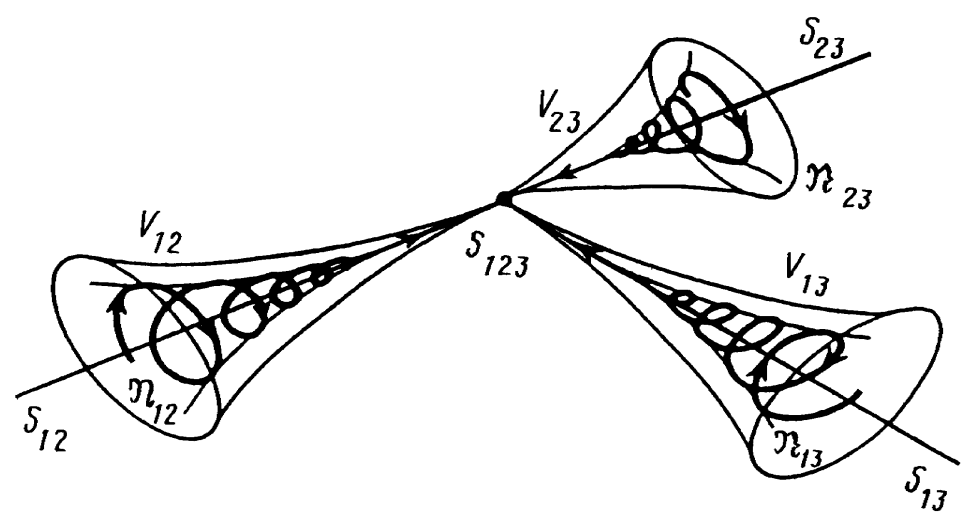

Рис. 3

Bсе сказанное остается справедливым mutatis mutandis при любой перестановке индексов $1,2,3$. 
Таким образом, оптимальный синтез для управляемой системы (28) объединяет в единой картине фазовые портреты, описанные в $\S \S 3$ и 4 . Роль пары траекторий, которая в инволютивном случае подходила к точке $w$ основного страта $W_{i j}$, играет проекция $k$-мерного слоя $\mathfrak{N}_{w}^{+}$, заполненная траекториями с учашающимися переключениями. Усложнение картины происходит в окрестности точек пересечения основных стратов. Кроме траекторий с учащающимися переключениями, которые лежат внутри пересекаюшихся стратов, имеются неособые траектории с учашаюшимися переключениями, на которых чередуются больше чем две вершины многогранника $U$. Набор этих вершин, порядок чередования и асимптотика времени чередования зависят от начальной точки траектории.

\section{Список литературы}

1. Bushaw D. W. Differential equations with a discontinuous forcing term. Princeton: Princeton Univ., Dept. of Math., 1952.

2. Фельдбаум А. А. Основы теории оптимальых автоматических систем. М.: Наука, 1966.

3. Болтлнский В. Г. Достаточные условия оптимальности // Докл. АН СССР. 1961. T. 140. № 5. C. 994-997.

4. Brunovsky P. Existence of regular synthesis for general control problems // J. Differential Equations. 1980. V. 38. №3. P. 317-343.

5. Зеликина Л. Ф. К вопросу о регулярном синтезе // Докл. АН СССР. 1982. Т. 267. №3. C. $532-535$.

6. Kupka I. The ubiquity of Fuller's phenomenon // Nonlinear controlllability and optimal control. Monograph Textbooks Pure Appl. Math / ed. H.Z. Sussman. № 133. New York: Dekker, 1990. P. 313-350.

7. Zelikin M., Borisov V. Theory of Chattering Control with Applications to Cosmonautics, Robotics, Economics, and Engineering. Boston: Birkhäuser, 1994.

8. Байтман М. М. Синтез оптимальных траекторий на плоскости. Рига: Зинатне, 1971.

9. Sussmann H. J. The structure of time-optimal trajectories for single input systems in the plane: the general real-analytic case // SIAM J. Control Optim. 1987. V. 25. P. 868-904.

10. Зеликина Л. Ф. Многомерный синтез и теоремы о магистрали в задачах оптимального управления // Вероятностные проблемы управления в экономике. М.: Наука, 1977. C. $33-114$.

11. Lobry C. Contrôlabilité des systèmes non linéaires // SIAM J. Control Optim. 1970. V. 8. P. 573-605.

12. Schättler $H$. On the local structure of time-optimal bang-bang trajectories in $\mathbb{R}^{3} / /$ SIAM J. Control Optim. 1988. V. 26. P. 186-204.

13. Krener A.J., Schättler $H$. The structure of small-time reachable sets in low dimensions // SIAM J. Control Optim. 1989. V. 27. P. 120-147.

14. Agrachev A., Kupka I., Gauthier J.-P. Generic Singularities of Sub-Riemannian metrics on $\mathbb{R}^{3}$ // C. R. Acad. Sci. Paris Sér. I Math. 1996. V. 322. P. 377-384.

15. Зеликин М.И., Борисов В. Ф. Режимы учащающихся переключений в задачах оптимального управления // Труды МИАН. 1991. Т. 197. С. 85-166.

16. Филиппов $A . \Phi$. Дифференциальные уравнения с разрывной правой частью // Матем. сб. 1960 . Т. 51 (93). № 1. С. 99-128.

17. Понтрягин Л. С., Болтянский В. Г., Гамкрелидзе Р. В., Мищенко Е. Ф. Математическая теория оптимальных процессов. М.: Физматгиз, 1961.

18. Зеликин М. И. Синтез оптимальных траекторий на пространствах представлений групп Ли // Матем. сб. 1987. Т. 132. № 4. С. 541-555.

19. Zelikin M. I. On the singular arcs // Problems Control Inform Theory. 1985. V. 14. № 2. P. $75-88$. 
20. Том Р. Локальные топологические свойства дифференцируемых отображений // Особенности дифференцируемых отображений. М.: Мир, 1968. С. 164-178.

21. Kelley H. J., Kopp R.E., Moyer H. G. Singular extremals // Topics in optimization / ed. G. Leitmann. New York: Acad. Press, 1967. P. 63-103.

22. Lewis R. M. Definitions of order and junction conditions in singular optimal control problems // SIAM J. Control Optim. 1980. V. 18. P. 21-32.

23. Манита Л. А. Поведение экстремалей в окрестности особых режимов и негладкие функции Ляпунова в задачах оптимального управления // Фундамент. и прикл. матем. 1996. T. 2. № 2. C. 449-485.

24. Борисов В. Ф. О числе предельных циклов фактор-системы $n$-мерной задачи Матем. сб. 1996. Т. 187. №12. С. 3-20.

Московский государственнњй

Поступила в редакцию университет им. М.В. Ломоносова;

10.06 .1998

Центральный экономико-математический институт РАН 